\title{
Identification of pathogen genomic variants through an integrated pipeline
}

\author{
Micah J Manary ${ }^{1,2^{*}}$, Suriya S Singhakul', Erika L Flannery' ${ }^{1}$, Selina ER Bopp ${ }^{3}$, Victoria C Corey ${ }^{1,2}$, \\ Andrew Taylor Bright ${ }^{1,2}$, Case W McNamara ${ }^{4}$, John R Walker ${ }^{4}$ and Elizabeth A Winzeler ${ }^{1}$
}

\begin{abstract}
Background: Whole-genome sequencing represents a powerful experimental tool for pathogen research. We present methods for the analysis of small eukaryotic genomes, including a streamlined system (called Platypus) for finding single nucleotide and copy number variants as well as recombination events.

Results: We have validated our pipeline using four sets of Plasmodium falciparum drug resistant data containing 26 clones from 3D7 and Dd2 background strains, identifying an average of 11 single nucleotide variants per clone. We also identify 8 copy number variants with contributions to resistance, and report for the first time that all analyzed amplification events are in tandem.

Conclusions: The Platypus pipeline provides malaria researchers with a powerful tool to analyze short read sequencing data. It provides an accurate way to detect SNVs using known software packages, and a novel methodology for detection of CNVs, though it does not currently support detection of small indels. We have validated that the pipeline detects known SNVs in a variety of samples while filtering out spurious data. We bundle the methods into a freely available package.
\end{abstract}

Keywords: Malaria, Sequencing, Genome, Polymorphism, Variant

\section{Background}

The detection of single nucleotide and copy number variants (SNVs and CNVs) conferring resistance to drug and vaccine candidates provides researchers with a powerful tool to choose the best combination of agents to treat infectious diseases such as malaria in specific regions, to study pathogen population dynamics and transmission, as well as to engineer new treatments that cannot be easily evaded. In addition, in organisms in which genetic complementation or backcrosses may be difficult or time consuming, whole genome sequencing (WGS) offers an opportunity to determine if second-site mutations may have been inadvertently introduced after transfection or transformation, and contribute to an observed phenotype.

\footnotetext{
* Correspondence: mmanary@ucsd.edu

'Department of Pediatrics, University of California, San Diego, School of Medicine, 9500 Gilman Drive 0741, La Jolla, California 92093, USA

${ }^{2}$ Biomedical Sciences Program, University of California, San Diego, 9500

Gilman Drive, La Jolla, California 92093, USA

Full list of author information is available at the end of the article
}

With the reduction in price and increased power of current short-read high-throughput WGS methods and the wide dispersal of a variety of sequencing platforms and accompanying support, full genome sequence data is now relatively easy to generate. Recent advances in the algorithmic and programmatic analysis of WGS data have led to a number of standards, especially the use of the Genome Analysis Toolkit (GATK) [1], being used in the analyses of human genomic data to detect SNVs and CNVs. However, there are opportunities for more comprehensive analyses of the genomes of simpler eukaryotes such as the $\sim 23.5 \mathrm{Mb}$ genome of Plasmodium falciparum, the apicomplexan parasite and etiological agent of human malaria, which has also served as a model for eukaryotic pathogen genomics since the completion and full assembly of its genome sequence in 2002 [2]. Full genome sequencing at $30-40 \mathrm{X}$ coverage is now readily achieved [3-6]. Such coverage allows for the identification of recombination events, the description of SNVs in sequences other than in the exomes, and the detection of small structural variants, including short-length insertion or deletion events. P. falciparum is responsible for up to a 
million deaths annually [7], and although its haploid genome is worthy of investigation for this reason alone, it also serves as an ideal test system because heterozygous calls generally do not need to be considered in sequence analysis validation (although mixed infections are a real concern) and a fully assembled reference genome is available [2]. Furthermore, the parasite can be sub-cloned and readily cultured in vitro within white-cell depleted, anucleated human erythrocytes [8], mitigating host DNA contamination.

In this manuscript, we introduce a validated pipeline for the comprehensive analysis of short-read WGS data in Plasmodium spp.. The pipeline, which can be readily adapted to other small eukaryotes, integrates well-known alignment tools and custom filtration options so that SNV or structural variant data can be easily generated and understood. We believe that the pipeline will work well, once adapted, with species of any ploidy (indeed, it has been used already in Arabidopsis analysis) and genomes of size up to $75 \mathrm{Mbp}$ have been tested. As well, we introduce improved algorithms for utilizing depth of coverage to call CNVs, improving on current GC bias normalization methods [9]. This pipeline is implemented in a stand-alone program called "Platypus", for open distribution and collaboration among research groups. We validate the pipeline using data from $26 \mathrm{P}$. falciparum samples with known SNVs and CNVs (Table 1), demonstrating both its accuracy and precision. This pipeline should allow those generating WGS data to not only find all SNVs and structural variants detected by other methods (as well as novel ones) but to eliminate all or almost all false positives, reducing ambiguity and potentially allowing WGS to substitute for complementation, Southern blotting, or other genetic methods designed to link phenotype to genotype.

\section{Implementation}

Current genotyping programs are generally designed to be conservative and as a consequence, return a large number of false positive variant calls. These programs, including GATK [1] and the sequence/alignment map toolbox (SAMTools) [13], typically allow the user to set a number of stringency filters such as the quality of the read alignment or bias towards a specific strand, that can theoretically be used to separate false from true positives. However, the actual threshold values for each filter are not pre-determined, and as such, it is left to the researcher to decide how to best utilize each metric, creating barriers for the novice user. Thus, we set out to create a set of empirically-derived filters for Plasmodium WGS data that could be used as a reference point for future SNV analyses.

To identify a robust set of filtering parameters we began with a list of 15,145 known SNVs identified using traditional Sanger resequencing of Dd2 to $7 \mathrm{X}$ coverage [14] and deposited in PlasmoDB (http://plasmodb.org) [15]. These distinguish the multidrug-resistant $P$. falciparum laboratory Indochina strain, Dd2, from the African drug-sensitive reference strain, 3D7. We then compared a $P$. falciparum Dd2 strain WGS short-read sequence obtained in our lab to the $P$. falciparum reference (3D7 strain) sequence. Our Dd2 sequence was generated with 70 bp paired-end reads on an Illumina Genome Analyzer II to a mean of $31 \mathrm{X}$ coverage with $96.4 \%$ of bases being covered by 5 reads or more. We considered the 15,145 curated SNVs to be true positives. All other SNVs detected were considered false positives, although it is likely that some of the novel SNVs are indeed true genetic differences (genetic diversity, especially in the subtelomeric regions, is extremely high approaching $90 \%$ diversity in at least one base position between field samples) [16]. We then worked to identify a set of filtering parameters, which would have the sensitivity to detect at least $90 \%$ of the known SNVs, while eliminating as many 'novel' SNVs as possible.

Because the entire mathematical domain of all commonly used filtering parameters (17 characteristics of SNVs and their combinations, see Table 2) is too large to search

Table 1 Whole-genome sequencing statistics

\begin{tabular}{cccccc}
\hline Experiment ID & Data source & Background strain & \# of genomes & Resistance & Gene conferring resistance \\
\hline Dd2 (parent) & {$[6,10-12]$} & $\mathrm{n} / \mathrm{a}$ & 1 & Chloroquine, Mefloquine, Pyrimethamine & $\mathrm{n} / \mathrm{a}$ \\
3D7 (parent) & {$[12]$} & $\mathrm{n} / \mathrm{a}$ & 1 & Sulfadoxine & $\mathrm{n} / \mathrm{a}$ \\
KAD707 and 458 & {$[12]$} & $\mathrm{Dd} 2$ & 4 & Imidazolopiperazine & $\mathrm{pfcarl}$ \\
CladoR & {$[10]$, this study } & $\mathrm{Dd} 2$ & 3 & Cladosporin & pfkrs1 \\
NITD609 (KAE609) & {$[6,11]$} & $\mathrm{Dd} 2$ & 3 & Spiroindolone & pfatp4 \\
NITD678 & {$[6,11]$} & $\mathrm{Dd} 2$ & 3 & Spiroindolone & pfatp4 \\
Evo & {$[6]$} & 3D7 & 14 & Atovaquone & mal_mito_3 \\
\hline
\end{tabular}

Whole genome sequencing data acquisition statistics from each of the experiments used to validate the pipeline. Note that all of the lines with variants to be called were sub-cloned before sequencing and were sequenced as 70 bp paired end reads, except the KAD (imidazolopiperazines resistant) lines, which were sequenced as $60 \mathrm{bp}$ single end reads. Library preparation details for sequences obtained from previous studies are available in their respective manuscripts. Library preparations for sequences obtained for this study (those resistant to cladosporin), are as follows: 200 bp fragments with 70 bp reads were prepared according to the manufacturers instructions using the Illumina NexTera XT sample preparation kit with accompanying primers and sheared using a Covaris E220x machine. $\mathrm{n} / \mathrm{a}$ - not applicable. 
Table 2 Optimized filtering parameters applied by Platypus

\begin{tabular}{lc}
\hline Filtering parameter & Optimized value \\
\hline Filters tested and found to affect specificity and sensitivity \\
Alignment aggregate mapping quality & $<7$ \\
Total quality & $<196.5$ \\
Depth of coverage & $<14$ \\
Strand bias Fisher's exact test & $>13.5$ \\
Filters tested and found not to affect specificity and sensitivity \\
Count of nucleotide identity & $n / a$ \\
Clipped read significance & $n / a$ \\
Depth of coverage per allele & $n / a$ \\
Quality by depth & $n / a$ \\
Homopolymer run & $n / a$ \\
Inbreeding coefficient & $n / a$ \\
Allele balance & $n / a$ \\
Confidence of elimination of incorrect genotype & $n / a$ \\
Root mean square of mapping quality & $n / a$ \\
Read position & $n / a$ \\
Spanning deletions & $n / a$ \\
Reads with mapping quality of zero & $n / a$ \\
Reads with a mapping quality of zero & $n / a$ \\
\hline
\end{tabular}

The final set of optimized filtering parameters evaluated by the searching algorithm. The set of informative filters gives $90 \%$ sensitivity with a specificity of $86 \%$.

exhaustively in efficient computational time, we developed a genetic searching optimization algorithm that searched over the entire domain of the 17 filtering parameters that characterize WGS data. This 'genetic' class of algorithm implements an objective function ('fitness') that is defined by several filters, and tries to minimize the value of the objective function over the entire domain (the lowest and highest possible values) of filters (every possible combination of filter choices) [17]. In our case we forced the sensitivity at various levels $(0-100 \%$ in increments of $0.5 \%$ ) and searched for the maximum specificity at each of those levels. Our objective function was a simple linear combination of all possible quality metrics for SNV data generated by GATK [1] and Picard (http://picard.sourceforge.net), with a varying polynomial coefficient matrix.

We chose a population of 10,000 parameter combinations to run through 100 evolutionary iterations. The algorithm we implemented included a low crossover rate $(0.5)$ and high mutation rate $(0.1)$ as well as a tournament pattern parental determination strategy with a tournament size of 100 , and with a guaranteed 10 elite children using the MatLab Global Optimization toolbox. These settings were dynamically determined to give consistency and robustness across a variety of sensitivity ranges. Iterating through a forced sensitivity level in $1 \%$ increments yielded a smooth progression along a similar combination of filtering parameters.

The list of 10,000 randomly chosen parameter combinations was assessed for both sensitivity and specificity. Each set of filtering parameters sorted the true positives into two categories ("called" or "not called") and similarly sorted the false positives; these calls were then evaluated for accuracy. Filtering sets that provided high specificity for a given level of sensitivity were carried over to the next round. The filtering parameters were then varied slightly within all successful sets, and individual parameters swapped between sets. After 100 iterative cycles, the most successful sets of filters converged on a single result - a theoretical optimal filtering set. We then added a further set of criteria based on the quality of the sequencing reads. The final optimized set excluded all SNV calls that met any of the following criteria listed in Table 2.

Using the optimal filtering set we detected $95.0 \%$ of the known SNVs with a specificity of $75.6 \%$ (8,315 total novel calls), and by lowering the total quality threshold we obtained a sensitivity of $90 \%$ of known SNVs, with a specificity of $85.1 \%$ (5,077 total novel calls). We generated a receiver operating characteristic curve (Figure 1) using optimal parameter sets at each sensitivity level, labeling three sensitivity threshold points of interest. Principal component analysis did not yield any statistically significant common genomic features (e.g. position on chromosome, position within gene, base pair transition) of the false positives detected, but 55\% (4,598 calls) were found in the $12 \%$ of the genome we define as sub-telomeric (within 100,000 bp of the chromosomal end) and which likely lie in regions that were not adequately covered by the 7X Dd2 Sanger sequencing [14]. We also searched over a space of multi-dimensional filters (those depending on more than one quality metric in a nonlinear relationship). This included all multiplicative combinations of two and three parameters, as well as exponential, power, logarithmic, and quadratic functions of single parameters, but these filters were unable to find results that were as good as a combined one-dimensional approach (that is, better than the optimal specificity and any sensitivity level), possibly due to the computational complexity of searching over multi-dimensional filters. This set of filtering parameters (Table 2) is thus implemented in our pipeline and was used for all subsequent analyses.

CNVs contribute substantially to drug resistance in Plasmodium and other eukaryotic pathogens [18-21]. The current methods for calling CNVs in Plasmodium spp. WGS data, like most pathogenic eukaryotes, rely on smoothing the depth of coverage data (e.g. number of reads aligned to the reference) [22-24]. Smoothing is needed because sequencing depends on multiple stochastic 


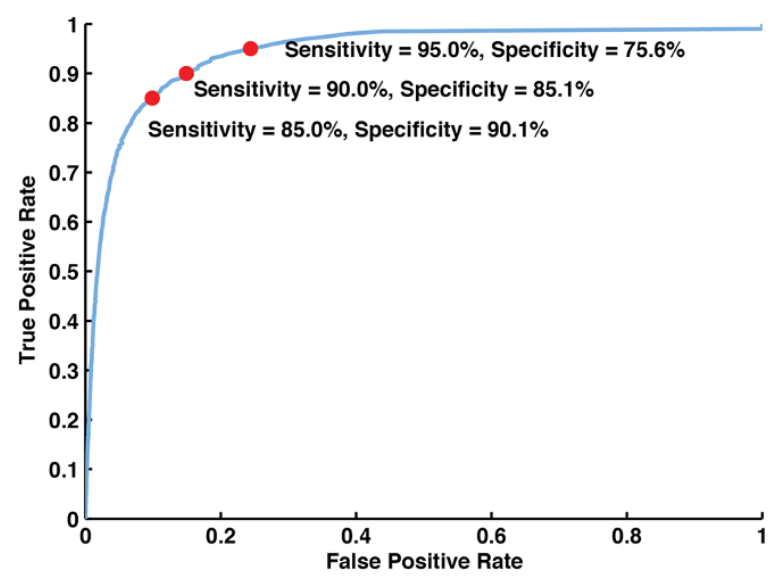

Figure 1 Receiver operating characteristic for SNV optimization. An ROC for the filtering parameters used in SNV detection optimization. Red points indicate the calculated specificity for certain fixed sensitivity levels. The blue line indicates the calculated specificity and sensitivity in $0.5 \%$ increments along the entire range of parameters. The optimal set of parameters for the GATK is given in Table 2. SNVs were then divided into either a 'called or 'not called' category based on these metrics and the current set of filters being used. The following metrics were obtained from the Unified Genotyper: read depth, strand bias, haplotype score, homopolymer run length, total positional mapping quality, number of null mapping qualities, total positional quality, total quality by depth, and genotype quality (DP, FS,

HaplotypeScore, HRun, MQ, MQ0, QUAL, QD, GQ). These SNVs were then hard filtered using the Variant Filtration Walker according to the given set of parameters, as in Table 2 .

processes and there can be great variability in the actual coverage over a given stretch of genomic DNA. Users are thus required to guess the appropriate smoothing parameters such as the number of base pairs to be averaged, meaning that the user already needs to know the approximate size of the CNV. Furthermore, it is known that there is also a non-stochastic bias in the depth of coverage due to the tendency of areas of high and low GC content to be sequenced less efficiently and this must also be accounted for, especially as P. falciparum is extremely AT-rich (81\%). Because we found that the current algorithms produced a large number of false calls when applied to our WGS data, we sought to address this problem by developing our own CNV calling algorithm.

The first improvement we made to the standard method was to improve GC bias correction. GC-content bias describes the dependence between fragment count (read coverage) and GC content found in high-throughput sequencing assays, particularly the Illumina Genome Analyzer technology. This bias can dominate the signal of interest for analyses that focus on measuring fragment abundance within a genome, such as copy number estimation and is not consistent between samples. We therefore analyzed regularities in the GC-bias patterns, and generated a closed-form compact description for this curve family. It is the GC content of the full DNA fragment (generally 100-200 bp), not only the sequenced read (the 50-100 bp sequenced on each end), that most influences this bias [9]. This GC bias distribution is unimodal: both GC rich fragments and AT rich fragments are under-represented in the sequencing results. Based on these findings, we devised a new method to calculate predicted coverage and correct for the bias.

In order to implement the correction we first calculate the GC content of the sequenced fragment (for paired end reads, both reads plus the insert) after aligning the reads to the reference genome (PlasmoDB 7.1 for imidazolopiperazines, 10.1 for the remainder), assuming that the insert matches the reference genome between the reads exactly. While this assumption does not account for potential point mutations, we have tested the effect of this on the bias normalization algorithm, and it is imperceptible, because point mutations occur so infrequently in a long insert size that the total GC content is not affected. After GC normalization CNVs become much more apparent than they were in the raw data (compare Figure $2 \mathrm{~A}$ and $\mathrm{B}$ ).

Secondly, after GC content normalization, the depth of coverage must be 'smoothed' so that true CNVs can be detected and random fluctuations in the data can be identified and discarded. However, smoothing algorithms tend to blur the divisions between otherwise sharp edges. Thus we also developed a simple iterative process of smoothing and edge detection that would identify these boundaries. This step integrates an iterative Weierstrass transform followed by edge detection through convolution with a first-derivative Gaussian kernel [25]. These can be described as follows: Let $D_{n}$ be the smoothed depth of coverage of a chromosome of length $l$ normalized after $n$ iterations. Then, with a Gaussian kernel $G$ with arbitrary coefficient $k$, we have, for all positions $j$ on the chromosome, $X_{j}$ :

$$
D_{n}=G * D_{n-1}
$$

Which we can expand to:

$$
D_{n}(\vec{x})=\sum_{i=1}^{l}\left(k_{1} e^{-k_{2}(\vec{x}-i) \cdot(\vec{x}-i)} D_{n-1}(i)\right)
$$

We can detect the edges of this data by finding all solutions:

$$
x \in l: \frac{\partial}{\partial x_{j}}\left(\frac{\partial G}{\partial x_{j}} * D_{n}\right)=0
$$

Which is expanded to: 


$$
x \in l: \sum_{i=1}^{l}\left(-2 k_{1} k_{2} e^{-k_{2}(\vec{x}-i) \cdot(\vec{x}-i)} D_{n}(i)+4 k_{1} k_{2}^{2}(x-i)^{2} e^{-k_{2}(\vec{x}-i) \cdot(\vec{x}-i)} D_{n}(i)\right)=0
$$

An example of the output of this algorithm is demonstrated in Figure 2C.

To save computational time, we applied the convolution theorem to take these operators in the Fourier space and as such, reduce all operations to point-wise multiplication. After each Weierstrass transform, edges are detected by the above formula. The total number of convolution iterations was set to be variable in the first in silico tests, ending only when no new edges had appeared in the last 10 iterations, but was eventually held constant at 5 because in practice no new edges appeared after the 2nd or 3rd iterations of the algorithm. We must treat the mitochondrial and apicoplast genome separately, as the depth of coverage of these is usually very different than the other Plasmodium chromosomes, even by an order of magnitude. The depth of coverage in each region (i.e. between each edge) is then compared to the sample mean, and those that are statistically higher or lower are assigned an amplification number based on their increase (or decrease) relative to the mean.

Recombination contributes substantially to the virulence of many eukaryotic pathogens such as P. falciparum and $T$. bruceii where genome encoded virulence factors are located in hyper-recombinogenic sections of the genome. In addition, such rearrangements could contribute to a phenotype if no causative SNV or clear dosage effect in a likely target is found. We thus sought to implement a program to find these recombination events.

Our strategy was to identify fragments with mated pairs that had abnormal insert sizes when they were aligned to a reference genome, especially ones with mated pairs that aligned to two different chromosomes or to vastly distant parts of the same chromosome. To find the initial events, all reads which had a mates aligning farther than $10 \mathrm{~kb}$ away from each other or on another chromosome were extracted and each region with a group of 10 or more overlapping reads with this property was de novo assembled using PRICE [26] with 20 cycles and otherwise standard settings (-icf \$x 115 , -nc 20, -dbmax 100, -maxHp 7, -lenf 2500 20). These contigs were then aligned against the reference genome using ClustalW to discover the origin of each part of the contig. Figure 3A, step IVc outlines the recombination detection process.

\section{Results and discussion}

To further test the validity of our optimized SNV filtration, and to test the accuracy of the predicted sensitivity (90\%) and specificity (85\%), we next evaluated the algorithm's ability to find rare mutations in isogenic lines created in our laboratory. We gathered WGS data from five separate chemical resistance experiments involving known and experimental antimalarial compounds (Table 1). In these experiments a clonal 3D7 or Dd2 line was exposed to sub-lethal concentrations of a small molecule inhibitor for 2-4 months. Parasites were then sub-cloned by limiting dilution and genomic DNA (gDNA) was sequenced, along with the gDNA of their isogenic parental clone. All strain genotype data were compared to the parent so that only genomic changes arising after chemical selection pressure would be identified. These strains have demonstrated resistances to a variety of small molecule inhibitors (atovaquone [6], cladosporin [10], spiroindolones [11] and imidazolopiperazines [12]). Most of these strains were initially characterized using a custom Affymetrix $P$. falciparum tiling microarray [28] and confirmed using Sanger sequencing or qPCR but some had been whole-genome sequenced previously, and some were sequenced specifically for this study (cladosporin resistant line). Altogether we analyzed data from 26 clones with their respective 2 parents. All were sequenced to $25-83 \mathrm{X}$ coverage using paired-end reads with $\sim 150 \mathrm{bp}$ fragment size on the Illumina Genome Analyzer II and Illumina HiSeq platforms (Tables 1 and 3).

For each of the 26 whole genome sequencing sample datasets, the Platypus pipeline was able to analyze and filter the sequencing data from tens of thousands of SNVs (examples of which are shown in Figure 4A) to a median of 6 final SNV calls (Figure 4B,C). The total number of raw SNVs, the number after filtering, and the number of these that were nonsynonymous for each of the experiments, are given in Table 3 . These results included $100 \%$ sensitivity concordance with the respective microarray and sequencing results, including all of the 20 unique SNVs that had been previously implicated in drug resistance (Table 3) as well as 127 additional mutations. More importantly, Platypus detected all SNVs that conferred the respective drug resistance phenotype and highlighted those which would cause a nonsynonymous change in an amino acid and alter protein function. Altogether, $63 \mathrm{SNVs}$ in the atovaquone, spiroindolone, and cladosporin resistant samples that were output by the Platypus program were confirmed as true hits by Sanger sequencing. In addition, $52 \mathrm{SNVs}$ in the atovaquone resistant samples that were closest to the cutoff line, but still excluded, were chosen as validation targets of a true negative result. These regions were sequenced and found to be insertion/deletion events, which our program is not currently designed to detect. 

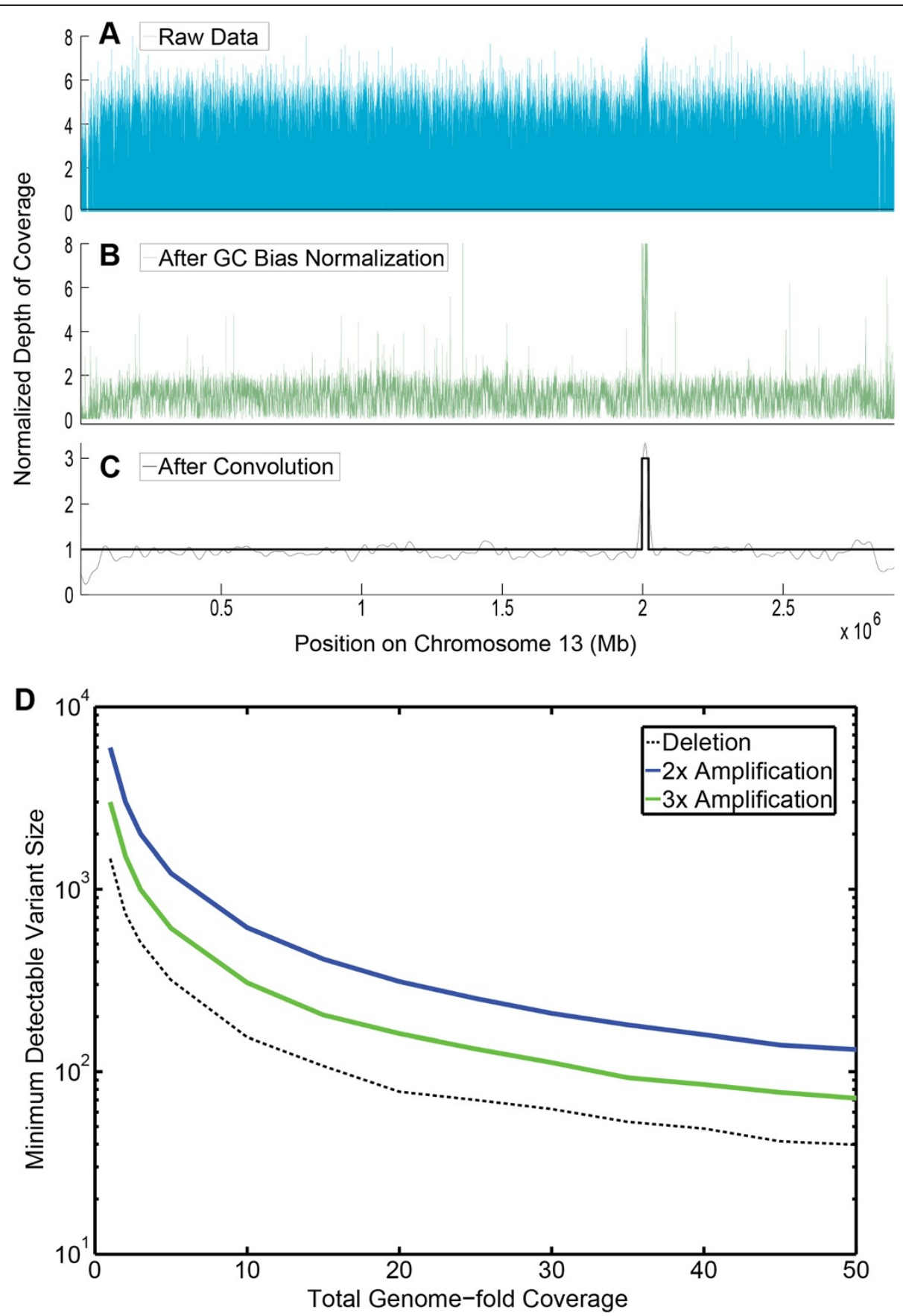

Figure 2 CNV detection in a cladosporin resistant Dd2 line. A. Raw depth of coverage data for chromosome 13 from the CladoR_clone3, which contains a microarray-verified amplification conferring resistance to cladosporin [10]. B. Depth of coverage normalized after GC bias correction. C. Normalized depth of coverage after the Platypus smoothing and edge-calling algorithm, with the actual copy number prediction overlaid. D. Theoretical minimum detectable variant size for a range of total genome coverage. Deletions are easier to detect and can be identified with less total coverage and in smaller sizes. Similarly, $3 x$ amplifications are easier to detect than $2 x$.

These results confirm that the program is robust and does not misinterpret sequencing data for Plasmodium spp.. In addition, the power of the program is clear - in each sample the Platypus filtered out everything (to the best of our knowledge) except that which was most relevant to the experiment. The time and effort saved by removing the long process of manual curation will have a major impact on the analysis of WGS data for Plasmodium and eukaryotic pathogen genomes in general. These methods can be directly applied to a variety of other pathogens as well, and we hope to see collaborative efforts to expand the pipeline to other organisms. 


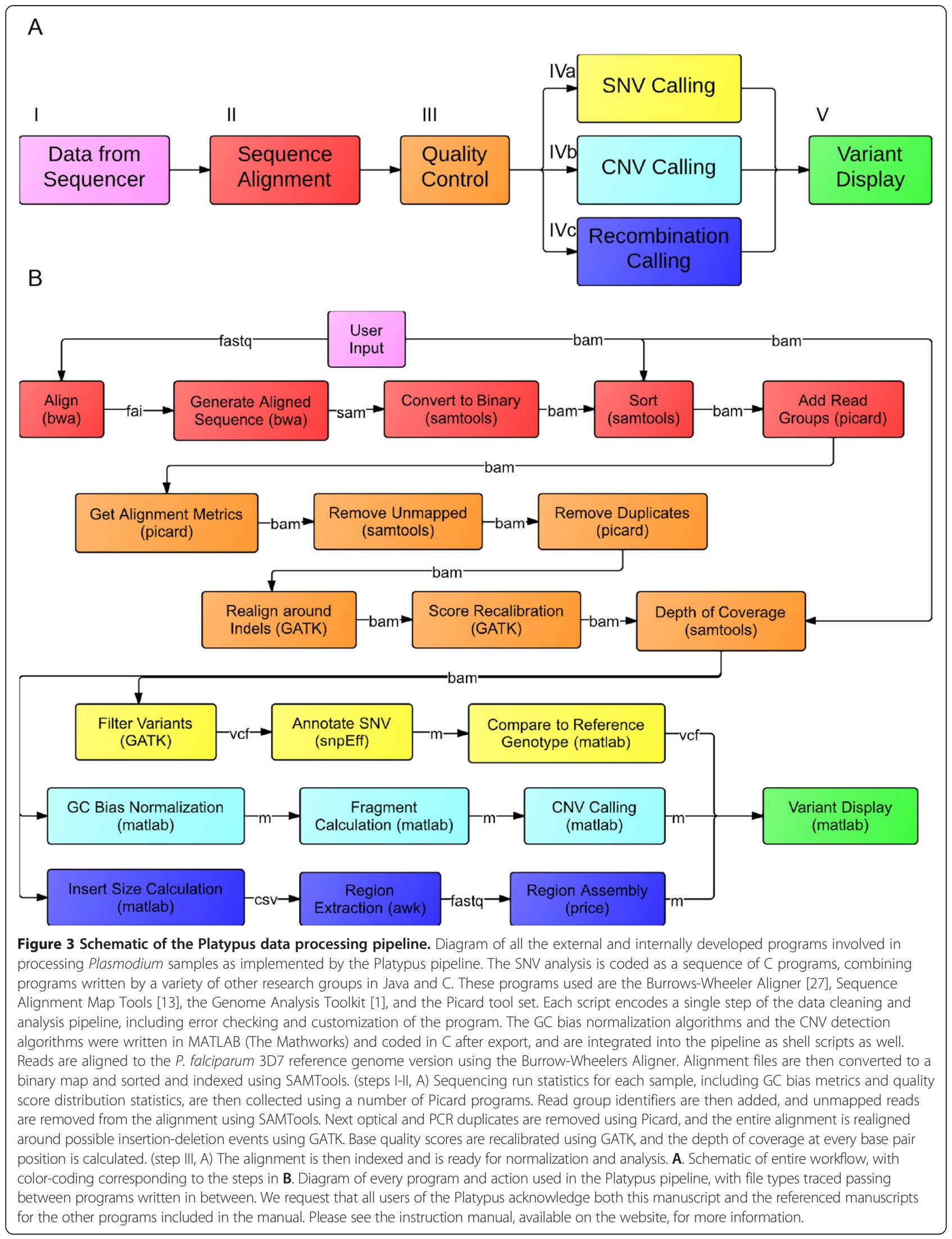


Table 3 Total number of SNVs detected using Platypus compared to simple filtering

\begin{tabular}{|c|c|c|c|c|c|}
\hline Experiment ID & \# Raw SNVs & \# SNVs from Q30 & \# SNVs from Platypus & $\begin{array}{l}\text { Amino acid change } \\
\text { conferring resistance }\end{array}$ & Genome total-fold coverage \\
\hline KAD707B1 & 613972 & 20 & 12 & L830V, M1069| & $43 x$ \\
\hline KAD707B2 & 50145 & 10 & 6 & M811, L830V & $62 x$ \\
\hline KAD707B3 & 52389 & 5 & 3 & L830V, S1076I & $50 x$ \\
\hline KAD458 & 60250 & 13 & 8 & S1076I, V1053A & $25 x$ \\
\hline CladoR_clone1 & 82488 & 53 & 32 & $\mathrm{n} / \mathrm{a}(\mathrm{CNV})$ & $67 x$ \\
\hline CladoR_clone2 & 100033 & 80 & 48 & $\mathrm{n} / \mathrm{a}(\mathrm{CNV})$ & $42 x$ \\
\hline CladoR_clone3 & 107611 & 68 & 41 & $\mathrm{n} / \mathrm{a}(\mathrm{CNV})$ & $47 x$ \\
\hline NITD609_1 & 50008 & 8 & 5 & I398F, P990R, CNV & $49 x$ \\
\hline NITD609_2 & 48201 & 12 & 7 & T418N, P990R & $48 x$ \\
\hline NITD609_3 & 48484 & 13 & 8 & D1247Y & $36 x$ \\
\hline NITD678_1 & 51665 & 18 & 11 & G223R & $39 x$ \\
\hline NITD678_2 & 56756 & 15 & 9 & A184S, P990R & $42 x$ \\
\hline NITD678_3 & 83190 & 55 & 33 & I203M, I263V & $44 x$ \\
\hline EvoR1a & 45388 & 8 & 5 & M133V & $83 x$ \\
\hline EvoR1a2 & 46151 & 12 & 7 & M133V & $76 x$ \\
\hline EvoR1b & 46462 & 10 & 6 & M133V & $60 x$ \\
\hline EvoR1b2 & 46377 & 15 & 9 & M133V & $79 x$ \\
\hline EvoR2a & 45565 & 3 & 2 & M133I & $64 x$ \\
\hline EvoR2a2 & 45604 & 3 & 2 & M133I & $67 x$ \\
\hline EvoR2b* & $n / a^{*}$ & $n / a^{*}$ & $\mathrm{n} / \mathrm{a}^{*}$ & $n / a^{*}$ & $1 x$ \\
\hline EvoR2b2 & 38188 & 3 & 2 & M133I & $82 x$ \\
\hline EvoR3a & 38224 & 7 & 4 & M133I & $54 x$ \\
\hline EvoR3b & 38620 & 7 & 4 & M133I & $62 x$ \\
\hline EvoR4a & 37404 & 5 & 3 & M133I, L144S & $67 x$ \\
\hline EvoR4b & 38333 & 5 & 3 & M133I, L144S & $78 x$ \\
\hline EvoR5a & 46563 & 3 & 2 & F267V & $79 x$ \\
\hline EvoR5b & 46890 & 3 & 2 & F267V & $65 x$ \\
\hline
\end{tabular}

*Sequencing data dominated by contamination.

Number of raw SNVs as well as the number of called SNVs from the Q30 metric [29] and the Platypus' final output. The Dd2 and 3D7 parents were compared to the 3D7 reference sequence from PlasmoDB v9.1, and then the drug resistant samples were compared to their respective parent. Results were compared to SNVs found by microarray or Sanger sequencing to assess sensitivity and specificity.

We note that the Platypus reduces the total number of SNVs from raw data by a factor of approximately $10^{3}-10^{4}$ (Table 3 ). While we cannot comprehensively genotype the tens of thousands of SNVs called initially by GATK or SAMTools, we have verified in atovaquone, spiroindolone, and cladosporin resistant lines that 63 of the SNVs called by Platypus are true genetic variants, and none of the 52 sites from the atovaquone resistant samples were excluded erroneously.

We also see (Table 3) that a comparison to the Q30 metric [29] identifies the Platypus as having significant gains over this simpler metric, reducing the number of SNVs called by a factor of approximately 1.6x. The sites called by the Q30 metric and excluded by the Platypus constituted 48 of the 52 sites that were
Sanger sequenced and subsequently discovered to be not true, validating their exclusion by the Platypus.

There is no standard set of filtering parameters to use with GATK, but we can compare to a set of published filter values for a comparable project [30]. Using Bright et al. as a comparison point, we can adapt their filters into our current pipeline. Doing so yields a $91 \%$ sensitivity level with a specificity of $45 \%$. We can see that these heuristically chosen values have a reasonable sensitivity threshold but do not hold up to empirically designed filters in terms of specificity.

The assessment of a false positive and false negative rate can of course never be perfected, but in all cases we have detected plausible drug resistance genes in all cases. Comparison with known values and with extensive 


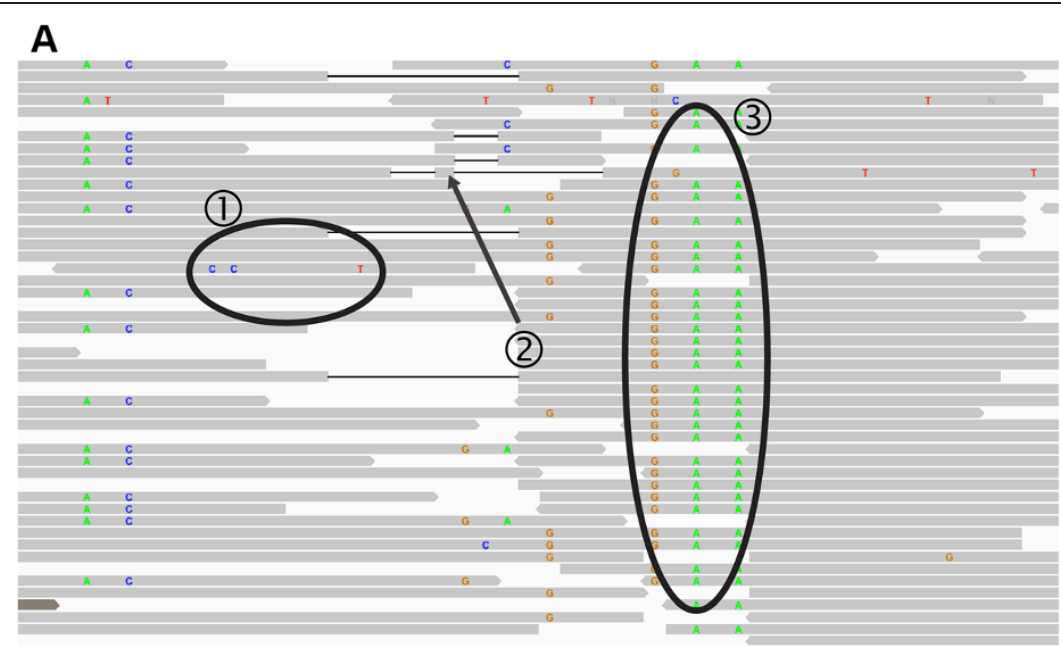

B

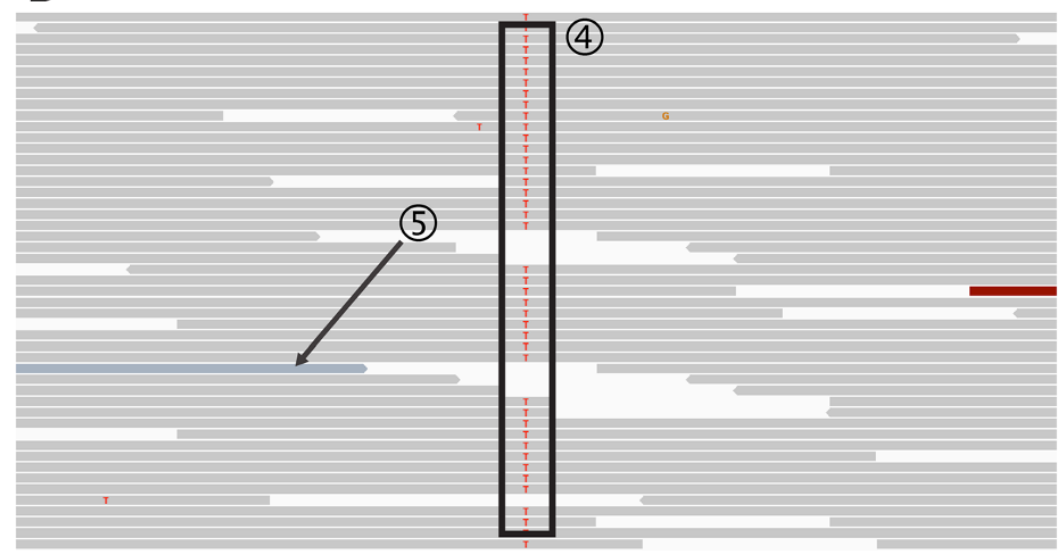

C

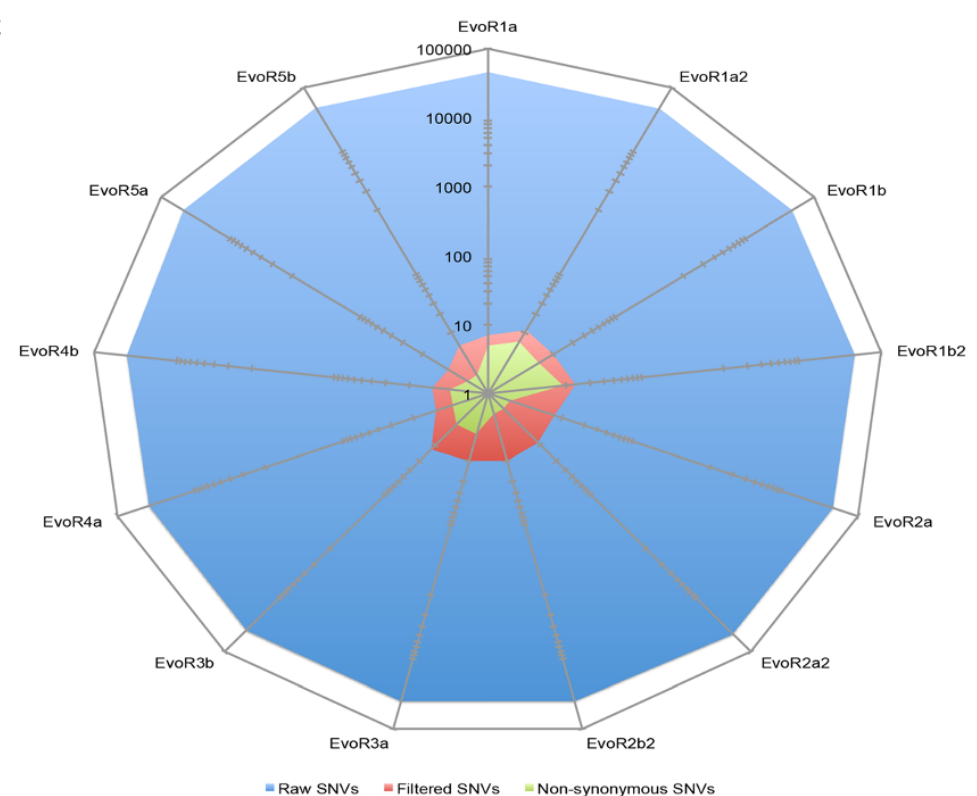

Figure 4 (See legend on next page.) 
(See figure on previous page.)

Figure 4 SNV filtering diagram for the Evo Samples. A. Read pileup of a region from EvoR1a2 including miscalled nucleotides within a read (1), misaligned reads (2 and 3), and false SNV calls due to a homopolymer run followed by a repetitive region. This region is an example of the false positive that the Platypus seeks to eliminate. B. Read pileup of a region from EvoR1a2 including a true SNV (4) but still including a number of misaligned reads (5). The Platypus seeks to extract these true positive SNVs from their surroundings. $\mathbf{C}$. Shows the number of raw SNV calls (blue) and the total number after filtration (red) and the subset of nonsynonymous SNVs (green) that may cause a change in the drug efficacy profile. Scale is logarithmic - the Platypus produces a $10^{3}-10^{4} \times$ reduction in SNV calls.

Sanger sequencing data confirms our calls, and even indicates that these sets of filters may be too lenient - that we may be detecting nonexistent SNVs rather than missing true ones.

To determine the sensitivity and specificity of the $\mathrm{CNV}$ detection algorithm, we sought to detect eight known amplifications from WGS data of the 26 strains with known structural variants, all of which had been discovered using our custom tiling microarray (Table 4). Figure 2A shows the raw depth of coverage data for a known amplification conferring cladosporin resistance on chromosome 13, and also indicates both the depth of coverage data after applying the detection algorithm (Figure 2B) and shows the copy number variant called from the data (Figure 2C).

Altogether Platypus identified all 8 unique CNVs that were known to exist in our strains. Our algorithm identified the large $\sim 100 \mathrm{~kb} C N V$ surrounding the $P$. falciparum multidrug resistance protein-1 gene ( $p f m d r 1$, PF3D7_0523000) in the 13 Dd2 derived strains [20,24] and the $5 \mathrm{~kb}$ GTP cyclohydrolase amplifications in 13 Dd2 (pfgch, PF3D7_1224000) derived strains as well as the smaller $1.6 \mathrm{~kb}$ amplification GTP cyclohydrolase in 13 3D7 derived strains [19]. We were also able to identify several independent larger amplifications that included lysyl tRNA synthetase (pfkrs1, PF3D7_1350100) in 3 strains that are resistant to cladosporin (a drug which targets lysyl-tRNA synthetase) [10], and an amplification on chromosome 1 in the EvoR5 strain that was grown in the presence of atovaquone, both confirmed by microarray as well [6]. We were also able to detect an amplification on chromosome 12 (containing pfatp4) in 3 of the spiroindolone resistant samples [6,11]. Although there was some ambiguity as to the number of copies (i.e. duplication or triplication), the Platypus also reported a SNV in one copy of pfatp 4 but not in the other copies of the gene. Furthermore, we discovered no spurious or novel amplification or deletion events, i.e. CNVs that were not detected by tiling microarray.

In addition, the boundaries for the respective $\mathrm{CNV}$ called by the algorithm for each sample correspond closely to the boundaries detected by microarray - the edges of the CNV algorithm lie, almost exclusively, within $100 \mathrm{bp}$ of the probes near the amplification boundaries. To further validate these data we also examined the read pileup near the predicted boundaries (Figure 5) for some cases (CladoR_clone1 shown). The read pileup allowed us to investigate the orientation of the amplification event. In the $\mathrm{Dd} 2$ lines mentioned above (those with detected amplifications surrounding pfmdr1, pfgch, and pfkrs1, pfatp4), 46-58\% of the paired-end reads at the beginning of each amplification mated to the end of that amplification with an abnormally large insert size and in the reverse read orientation (Figure 5, inset). This indicates that the amplification event is not only on the same chromosome but also in tandem - that is, the amplified genomic region is adjacent to the originating sequence (Table 4). Interestingly, we found all amplifications that we examined were in a tandem orientation.

To test the theoretical limits of the CNV detection algorithm we also generated simulated amplifications

Table 4 CNVs detected using WGS and genomic microarrays

\begin{tabular}{|c|c|c|c|c|c|}
\hline Strain & Position (Micr.) & Position (Seq.) & Presumed relevant gene & Copy number (Seq.) & Orientation (Seq.) \\
\hline all 3D7 derived lines & chr12_974243-975980 & chr12_974276-976007 & pfgch & 2 & tandem \\
\hline all Dd2 derived lines & chr5_892863-968421 & chr5_892872-968429 & pfmdr1 & 2 & tandem \\
\hline all Dd2 derived lines & chr12_970985-975864 & chr12_971011-975866 & pfgch & 2 & tandem \\
\hline Evo5a/b & chr1_428540-643352 & chr1_428538-643350 & pfmrp 1 & 2 & unknown* \\
\hline CladoR_clone1 & chr13_1996541-2018534 & chr13_1996635-2018727 & pfkrs 1 & 3 & tandem \\
\hline CladoR_clone2 & chr13_1996668-2055132 & chr13_1996621-2055107 & pfkrs 1 & 3 & tandem \\
\hline CladoR_clone3 & chr13_2001466-2051201 & chr13_2001482-2051233 & pfkrs 1 & 3 & tandem \\
\hline NITD678_clone1 & chr12_510069-633784 & chr12_510123-633834 & pfatp4 & $2-3$ & tandem \\
\hline
\end{tabular}

*Orientation could not be determined as the amplification continued into the telomeric region.

Predicted CNV calls with position from both WGS (Seq.) using Platypus and microarray data (Micr.), with predicted copy number compared to the 3D7 reference and orientation of the amplification as determined by Platypus. 


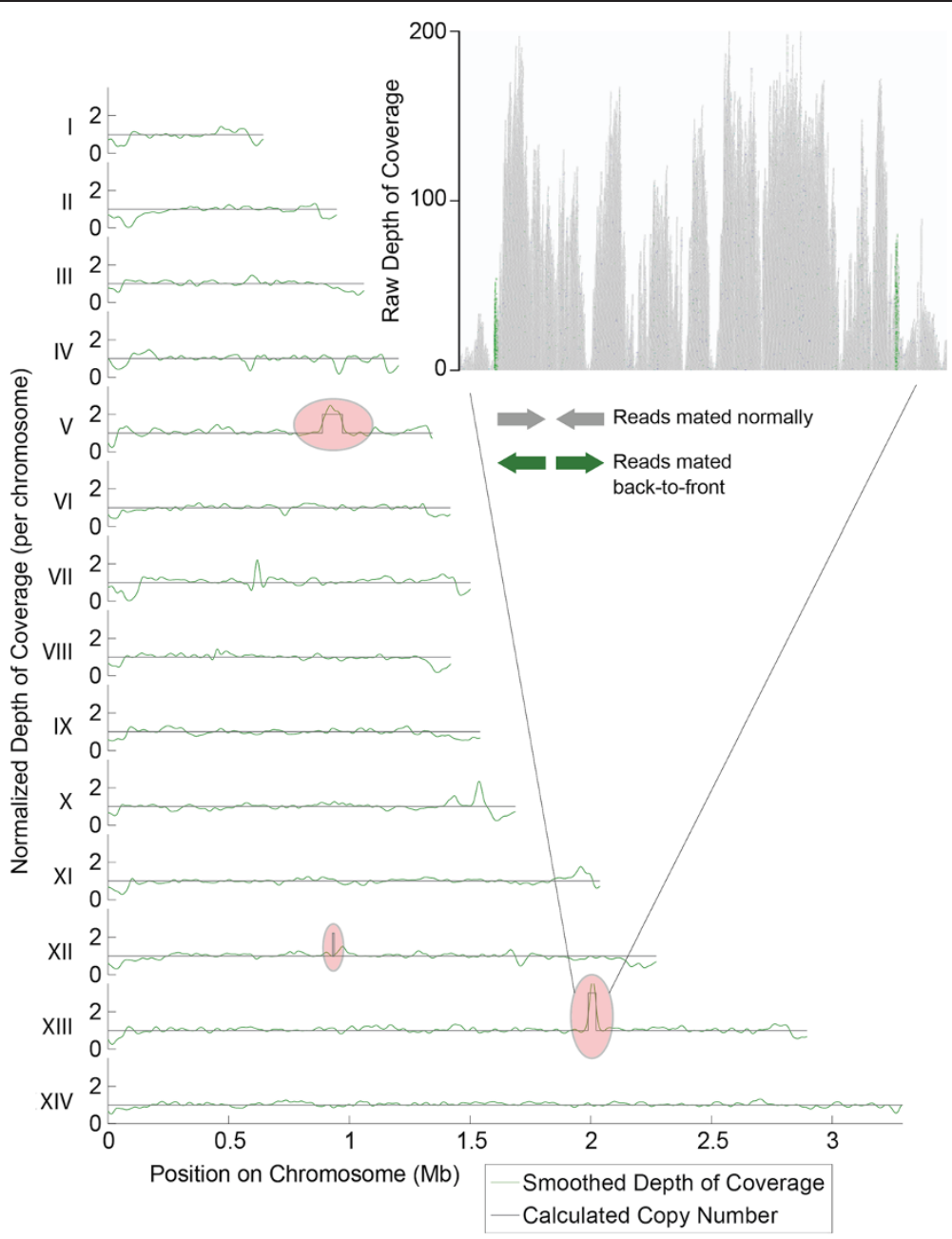

Figure 5 Detecting genome amplification events. Depth of coverage data for all 14 chromosomes of a Dd2 clonal line with evolved resistance to cladosporin (CladoR_clone3) [10]. The curve represents the output of the CNV detection algorithm, while the black line is the actual copy number call by the program. The two visible amplifications detected are the one encompassing the pfmdr locus on chromosome 5 and the amplification associated with cladosporin-resistance on chromosome 13 [10]. A third amplification on chromosome 12 containing pfgch is visible at higher magnification. The inset shows a read pileup for the region containing an amplification on chromosome 13. The green colored reads indicate those with an abnormal insert size with mated reads mapping back-to-back rather than front-to-front. This is evidence that the amplification is in tandem with the original copy on the chromosome.

and deletions by producing a depth of coverage from a random distribution that had a mean twice the sample mean (amplifications), or by completely eliminating certain reads (deletions). Deletions could be detected with low coverage and in very small sizes, with a theoretical limit around 3x coverage and $500 \mathrm{bp}$ (with $100 \mathrm{bp}$ reads). Amplifications were harder to detect, but the limit is approximately 1000 bp at 20x total genomic coverage. This limit is accurate for all deletions and for amplifications up to four times, after which point the algorithm can only detect the presence of a variant and estimate its copy number (an estimate of $6 x$ indicates a $5 x-7 x$ CNV). Figure $2 \mathrm{D}$ demonstrates the theoretical capabilities of the algorithm in detecting both deletion and amplification events for various total genome coverage levels.
We compared our $\mathrm{CNV}$ calling algorithm to BreakDancer, a similar program used to detect both copy number and recombination events using the default [31]. Using a set of parameters equivalent to those published in Chen et al., (4 standard deviation threshold, Q $>39, M Q>35$ ) we see that BreakDancer is fully able to detect all CNVs present in our samples (those detected by microarray and/or whole genome sequencing), but it also identifies 73 other CNVs ranging from $434 \mathrm{bp}$ to $11639 \mathrm{bp}$ that we do not detect by any method. Indeed, qPCR amplification of these speculated regions indicates no change in copy number in any of these regions not detected by other high throughout methods.

Our algorithm identified 15 of these potential interchromosomal events in 13 3D7 Evo strains that resulted 
in partial gene deletion/duplication paired events, which also registered as deletion/amplification events in the $\mathrm{CNV}$ algorithm. In order to validate that these were indeed recombination events, the PRICE assembler was seeded with a single read from the suspected recombination region, and then de novo assembly was carried out using all reads that surrounded the alignment of the seeded read and its mate pair, as well as their respective mates. Altogether we were able to validate all 15 of these likely recombination events despite the hypervariability and high AT content (as high as 95\% in these regions) that makes the specificity and uniqueness of these sequences very low. Figure 6 shows the sequence of one of the predicted regions as well as the matching sequence of the PCR product. To seek further evidence of genomic rearrangement, the 15 reassembled recombination events were validated by Sanger sequencing using primers that spanned the region. We found that the sequence of the de novo assembled fragment matched that of the Sanger sequencing product to within a one base pair difference in all 15 events. These assemblies spanned chromosomal sub-telomeric regions, and spliced together related genes. The PCR sequences of these assemblies and their clustal alignments are included in the supplemental information, and the mapping of each recombination event to each isolate is included in the supplement (see Additional file 1: Table S1). BLAST/CLUSTALOmega analysis verifies that these regions map to two separate chromosomes as expected.

\section{Conclusions}

A problem with using WGS is that it may be inaccessible to laboratories that are not strong in bioinformatics. To address this issue we integrated these modules into a program that we call Platypus (Figure 3 ). The pipeline integrates a number of other software programs, and these are referenced in full in this manuscript and in the software documentation. Platypus takes as input either unaligned FastA/FastQ sequencing data, or aligned data in the BAM format. SNVs, CNVs, and potential recombination events are output as annotated text files which can be cross-referenced with PlasmoDB or similar databases.

The Platypus pipeline provides malaria researchers with a powerful tool to analyze short read sequencing data. It provides an accurate way to detect SNVs using known software packages, and a novel methodology for detection of CNVs, though it does not currently support detection of small indels. We have validated that the pipeline detects known SNVs in a variety of samples while filtering out spurious data. We have also tested it against both computational samples and actual data with known CNVs (both deletions and amplifications as verified by microarray) and it can detect the size and boundaries of these CNVs with a high degree of accuracy. The success of the Platypus software in both detecting real genetic variants and avoiding the reporting of false positives over a number of parasite lines can be attributed to its basis on first principles. The SNV detection was

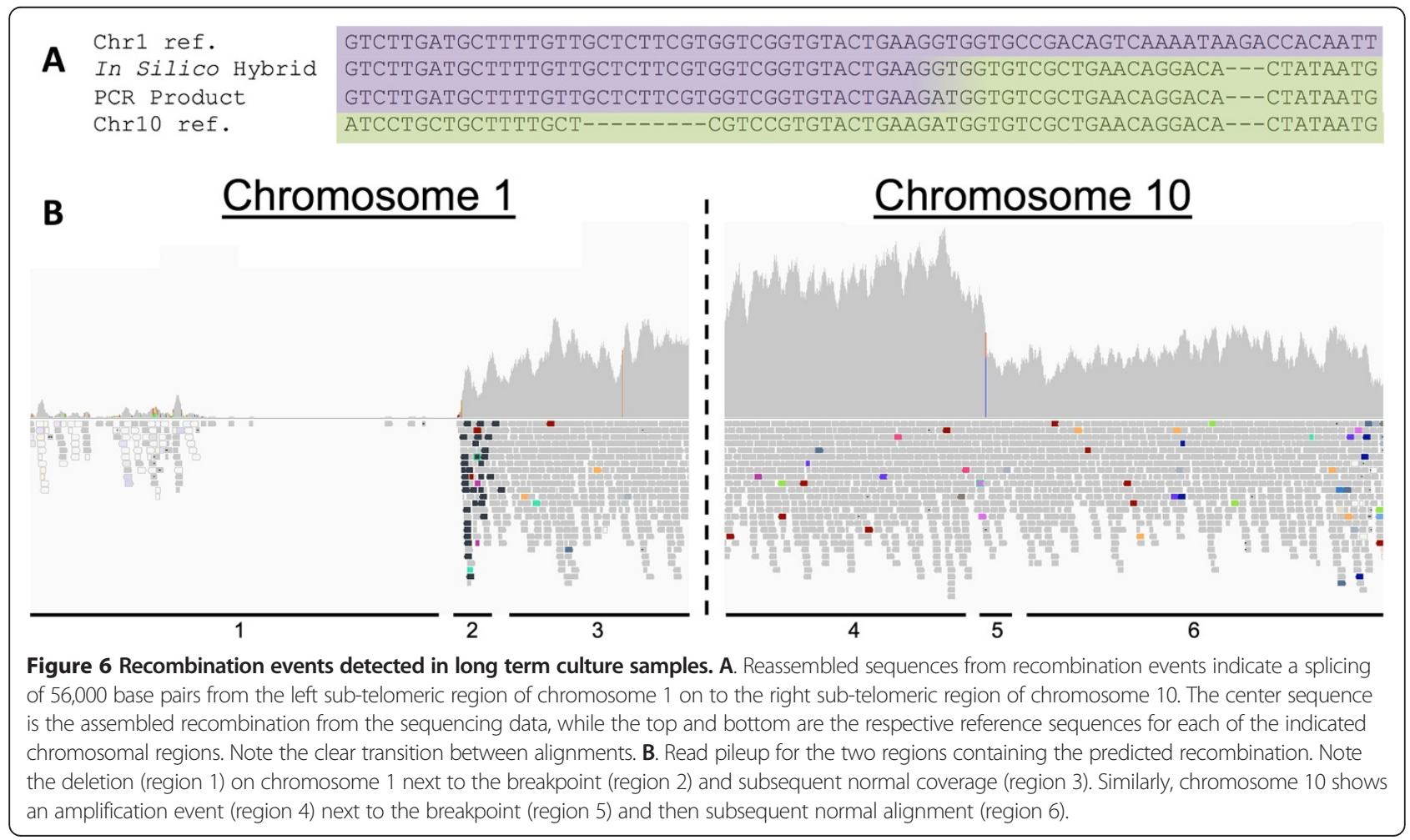


specifically designed only to use filters that accurately segregated true and false positives, and the robustness of this approach is evident, as there is a completely smooth transition between sensitivity/specificity levels when varying over the ideal filter set. The CNV detection was based on the fundamental theorem of digital signal processing, and indeed the assumptions of this field applies directly to the signals coming off a next-generation whole genome sequencer, complete with random and systematic biases. This streamlined package offers an initial starting point for the field to analyze and report these data in a consistent manner.

\section{Availability and requirements}

The program is platform independent and can be run on ordinary desktop computers: In our case all analysis and computer programming was done using Mac OSX 10.7.3 on a Mac Pro with 12 multi-threaded processors on 2 cores and 32Gb of $1066 \mathrm{MHz}$ DDR2 RAM. Altogether 24Gb of RAM was made available to Java while the Platypus was running. We have made Platypus freely available as an open-source package at <http://sourceforge.net/projects/ platypusmga/>.

\section{Additional file}

Additional file 1: Table S1. Presence (1) or absence (0) of potential recombination events in various isolates from the evolution experiments (Bopp et al, 2013) [6].

\section{Abbreviations}

Bp: Base pair; CNV: Copy number variant; DNA: Deoxyribonucleic acid; FastA: Fast (all) format; FastQ: Fast (all) quality format; GATK: Genome analysis toolkit; gDNA: Genomic DNA; Mb: Mega- base pair; PlasmoDB: Plasmodium database; Platypus: Pathogen lovers automated type uncovering software; SAM: Sequence alignment map; SNV: Single nucleotide variant; spp: Species pluralis; WGS: Whole genome sequencing.

\section{Competing interests}

The authors declare that they have no competing interests.

\section{Authors' contributions}

MJM designed the program, aligned the sequences, wrote the computer algorithms, ran the validations, performed the human analysis, and wrote the initial drafts. SSS performed human analysis and ran validation experiments. ELF provided extensive manuscript preparation and program testing. SERB cultured parasites and analyzed the data for the evolution experiment. VCC assisted in program validations and aligning sequences and assisted in manuscript preparation. ATB assisted in the program development and validation. CWM cultured parasites and assisted in the analysis of the cladosporin experiment. JRW sequenced all the samples and assisted in the initial analysis. EAW helped design the program, assisted in validation strategies, and assisted with writing the manuscript. All authors read, reviewed, critiqued, and approved the full manuscript.

\section{Acknowledgements}

The authors would like to acknowledge Stephan Meister and Shinji Okitsu for advice on the manuscript. This work was supported by 7 R01 Al090141-02 and by the Bill and Melinda Gates Foundation (OPP1054480) and MJM was supported by funding from the University of California San Diego Medical Scientist Training Program, the University of California San Diego Genetics Training Grant, and fellowship from the Hertz Foundation.

\section{Author details}

'Department of Pediatrics, University of California, San Diego, School of Medicine, 9500 Gilman Drive 0741, La Jolla, California 92093, USA.

${ }^{2}$ Biomedical Sciences Program, University of California, San Diego, 9500 Gilman Drive, La Jolla, California 92093, USA. ${ }^{3}$ Immunology and Infectious Diseases, Harvard School of Public Health, 665 Huntington Avenue, Boston, Massachusetts 02115, USA. ${ }^{4}$ Genomics Institute of the Novartis Research Foundation, 10675 John Jay Hopkins Drive, San Diego, California 92121, USA.

\section{Received: 9 December 2013 Accepted: 6 February 2014}

Published: 3 March 2014

\section{References}

1. McKenna A, Hanna M, Banks E, Sivachenko A, Cibulskis K, Kernytsky A, Garimella K, Altshuler D, Gabriel S, Daly M, DePristo MA: The Genome analysis toolkit: a MapReduce framework for analyzing next-generation DNA sequencing data. Genome Res 2010, 20:1297-1303.

2. Gardner MJ, Hall N, Fung E, White O, Berriman M, Hyman RW, Carlton JM, Pain A, Nelson KE, Bowman S, et al: Genome sequence of the human malaria parasite Plasmodium falciparum. Nature 2002, 419:498-511.

3. Manske M, Miotto O, Campino S, Auburn S, Almagro-Garcia J, Maslen G, O'Brien J, Djimde A, Doumbo O, Zongo I: Analysis of Plasmodium falciparum diversity in natural infections by deep sequencing. Nature 2012, 487(7407):375-379.

4. Miotto O, Almagro-Garcia J, Manske M, Macinnis B, Campino S, Rockett KA, Amaratunga C, Lim P, Suon S, Sreng S, et al: Multiple populations of artemisinin-resistant Plasmodium falciparum in Cambodia. Nat Genet 2013, 45(6):648-655.

5. Samarakoon U, Regier A, Tan A, Desany BA, Collins B, Tan JC, Emrich SJ, Ferdig MT: High-throughput 454 resequencing for allele discovery and recombination mapping in Plasmodium falciparum. BMC Genomics 2011, 12:116.

6. Bopp SER, Manary MJ, Bright AT, Johnston GL, Dharia NV, Luna FL, McCormack S, Plouffe D, McNamara CW, Walker JR, et al: Mitotic evolution of Plasmodium falciparum shows a stable core Genome but recombination in antigen families. PLoS Genet 2013, 9:e1003293.

7. Murray CJ, Rosenfeld LC, Lim SS, Andrews KG, Foreman KJ, Haring D, Fullman N, Naghavi M, Lozano R, Lopez AD: Global malaria mortality between 1980 and 2010: a systematic analysis. Lancet 2012, 379:413-431.

8. Trager W, Jensen JB: Human malaria parasites in continuous culture. Science 1976, 193:673-675.

9. Benjamini Y, Speed T: Estimation and correction for GC-content bias in high throughput sequencing. Berkeley, CA USA: Tech Rep; 2011.

10. Hoepfner D, McNamara CW, Lim CS, Studer C, Riedl R, Aust T, McCormack SL, Plouffe DM, Meister S, Schuierer S, et al: Selective and specific inhibition of the plasmodium falciparum lysyl-tRNA synthetase by the fungal secondary metabolite cladosporin. Cell Host Microbe 2012, 11:654-663.

11. Rottmann M, McNamara C, Yeung BK, Lee MC, Zou B, Russell B, Seitz P, Plouffe DM, Dharia NV, Tan J, et al: Spiroindolones, a potent compound class for the treatment of malaria. Science 2010, 329:1175-1180.

12. Meister S, Plouffe DM, Kuhen KL, Bonamy GM, Wu T, Barnes SW, Bopp SE, Borboa R, Bright AT, Che J, et al: Imaging of Plasmodium liver stages to drive next-generation antimalarial drug discovery. Science 2011, 334:1372-1377.

13. Li H, Handsaker B, Wysoker A, Fennell T, Ruan J, Homer N, Marth G, Abecasis G, Durbin R, Subgroup GPDP: The sequence alignment/map format and SAMtools. Bioinformatics 2009, 25:2078-2079.

14. Volkman SK, Sabeti PC, DeCaprio D, Neafsey DE, Schaffner SF, Milner DA Jr, Daily JP, Sarr O, Ndiaye D, Ndir O, et al: A genome-wide map of diversity in Plasmodium falciparum. Nat Genet 2007, 39:113-119.

15. Aurrecoechea C, Brestelli J, Brunk BP, Dommer J, Fischer S, Gajria B, Gao X, Gingle A, Grant G, Harb OS: PlasmoDB: a functional genomic database for malaria parasites. Nucleic Acids Res 2009, 37:D539-D543.

16. Barry AE, Leliwa-Sytek A, Tavul L, Imrie H, Migot-Nabias F, Brown SM McVean GA, Day KP: Population genomics of the immune evasion (var) genes of Plasmodium falciparum. PLoS Pathog 2007, 3:e34

17. Weise T: Global optimization algorithms-theory and application. La Jolla, CA USA: Self-Published; 2009

18. Nair S, Miller B, Barends M, Jaidee A, Patel J, Mayxay M, Newton P, Nosten F, Ferdig MT, Anderson TJ: Adaptive copy number evolution in malaria parasites. PLoS Genet 2008, 4:e1000243. 
19. Kidgell C, Volkman SK, Daily J, Borevitz JO, Plouffe D, Zhou Y, Johnson JR, Le Roch K, Sarr O, Ndir O, et al: A systematic map of genetic variation in Plasmodium falciparum. PLoS Pathog 2006, 2:e57.

20. Wilson CM, Serrano AE, Wasley A, Bogenschutz MP, Shankar AH, Wirth DF: Amplification of a gene related to mammalian mdr genes in drug-resistant Plasmodium falciparum. Science 1989, 244:1184-1186.

21. Singh A, Rosenthal PJ: Selection of cysteine protease inhibitor-resistant malaria parasites is accompanied by amplification of falcipain genes and alteration in inhibitor transport. J Biol Chem 2004, 279:35236-35241.

22. Medvedev P, Fiume M, Dzamba M, Smith T, Brudno M: Detecting copy number variation with mated short reads. Genome Res 2010, 20:1613-1622.

23. Yoon S, Xuan Z, Makarov V, Ye K, Sebat J: Sensitive and accurate detection of copy number variants using read depth of coverage. Genome Res 2009, 19:1586-1592.

24. Robinson T, Campino SG, Auburn S, Assefa SA, Polley SD, Manske M, Maclnnis B, Rockett KA, Maslen GL, Sanders M: Drug-resistant genotypes and multi-clonality in Plasmodium falciparum analysed by direct genome sequencing from peripheral blood of malaria patients. PLoS One 2011, 6:e23204.

25. Smith SW: The Scientist and Engineer's Guide to Digital Signal Processing, 1999. PO Box: California Technical Publishing; 2009.

26. Ruby JG, Bellare P, DeRisi JL: PRICE: Software for the targeted assembly of components of (Meta) Genomic sequence data. G3 (Bethesda) 2013, 3:865-880.

27. Li H, Ruan J, Durbin R: Mapping short DNA sequencing reads and calling variants using mapping quality scores. Genome Res 2008, 18:1851-1858.

28. Dharia NV, Sidhu AB, Cassera MB, Westenberger SJ, Bopp SE, Eastman RT, Plouffe D, Batalov S, Park DJ, Volkman SK, et al: Use of high-density tiling microarrays to identify mutations globally and elucidate mechanisms of drug resistance in Plasmodium falciparum. Genome Biol 2009, 10:R21.

29. Neafsey DE, Galinsky K, Jiang RH, Young L, Sykes SM, Saif S, Gujja S, Goldberg JM, Young S, Zeng Q, Chapman SB, Dash AP, Anvikar AR, Sutton PL, Birren BW, Escalante AA, Barnwell JW, Carlton JM: The malaria parasite Plasmodium vivax exhibits greater genetic diversity than Plasmodium falciparum. Nat Genet 2012, 44(9):1046-50.

30. Dharia NV, Bright AT, Westenberger SJ, Barnes SW, Batalov S, Kuhen K, Borboa R, Federe GC, McClean CM, Vinetz JM, Neyra V, Llanos-Cuentas A, Barnwell JW, Walker JR, Winzeler EA: Whole-genome sequencing and microarray analysis of ex vivo Plasmodium vivax reveal selective pressure on putative drug resistance genes. Proc Natl Acad Sci U S A 2010, 107(46):20045-50.

31. Chen K, Wallis JW, McLellan MD, Larson DE, Kalicki JM, Pohl CS, McGrath SD, Wendl MC, Zhang Q, Locke DP: BreakDancer: an algorithm for high-resolution mapping of genomic structural variation. Nat Methods 2009, 6:677-681.

doi:10.1186/1471-2105-15-63

Cite this article as: Manary et al.: Identification of pathogen genomic variants through an integrated pipeline. BMC Bioinformatics 2014 15:63.

\section{Submit your next manuscript to BioMed Central and take full advantage of:}

- Convenient online submission

- Thorough peer review

- No space constraints or color figure charges

- Immediate publication on acceptance

- Inclusion in PubMed, CAS, Scopus and Google Scholar

- Research which is freely available for redistribution 